$\mathrm{DE}$

M E D I C I N A

T R O P I C A L

$\mathrm{DE}$

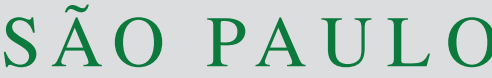

JOURNAL OF THE SÃO PAULO INSTITUTE OF TROPICAL MEDICINE

'Universidade de São Paulo, Instituto de Medicina Tropical de São Paulo, São Paulo, São Paulo, Brazil

${ }^{2}$ Instituto de Assistência Médica ao Servidor Público Estadual de São Paulo, São Paulo, Brazil

${ }^{3}$ University of Leicester, Leicester, United Kingdom

${ }^{4}$ Hospital Totalcor, São Paulo, São Paulo, Brazil

Correspondence to: Silvia Figueiredo

Costa

Universidade de São Paulo, Instituto de Medicina Tropical de São Paulo,

Av. Dr. Eneias Carvalho de Aguiar, 255,

CEP 05403000, São Paulo, SP, Brazil

E-mail: silviacosta@usp.br

Received: 21 August 2020

Accepted: 5 April 2021

\section{Colistin-resistant Escherichia coli belonging to different sequence types: genetic characterization of isolates responsible for colonization, community- and healthcare- acquired infections}

\author{
Yrving Paiva', Debora Satie Nagano ${ }^{\circledR} 1$, Andre Luis Franco Cotia ${ }^{1}$, Thais \\ Guimarães ${ }^{2}$, Roberta Cristina Ruedas Martins' ${ }^{1}$, Lauro Vieira Perdigão Neto', \\ Marina Farrel Côrtes ${ }^{1}$, Ana Paula Marchi', Louise Corscadden ${ }^{3}$, Anna Silva \\ Machado $^{4}$, Alexandre Inácio de Paula², Lucas Augusto Moyses Franco ${ }^{(1,}$, \\ Patricia Regina Neves ${ }^{1}$, Anna Sara Levin' ${ }^{1}$, Silvia Figueiredo Costa ${ }^{\circledR}$
}

\section{ABSTRACT}

The plasmid-mediated colistin-resistance gene named $\mathrm{mcr}-1$ has been recently described in different countries and it became a public health challenge. Of note, few studies have addressed the spread of Escherichia coli harboring the $m c r-1$ gene in both, community and hospital settings. A total of seven colistin-resistant $E$. coli carrying $m c r-1$, collected from 2016 to 2018 , from community $(n=4)$, healthcare-acquired infections $(n=2)$ and colonization $(n=1)$ were identified in three high complexity hospitals in Sao Paulo, Brazil. These colistinresistant isolates were screened for $m c r$ genes by PCR and all strains were submitted to Whole Genome Sequencing and the conjugation experiment. The seven strains belonged to seven distinct sequence types (ST744, ST131, ST69, ST48, ST354, ST57, ST10), and they differ regarding the resistance profiles. Transference of $m c r-1$ by conjugation to $E$. coli strain $\mathrm{C} 600$ was possible in five of the seven isolates. The $m c r-1$ gene was found in plasmid types IncX4 or IncI2. Three of the isolates have ESBL-encoding genes $\left(b l a_{\text {СТХ-М-2 }}, \mathrm{n}=2 ; b l a_{\text {СТХ-М-8 }}, \mathrm{n}=1\right)$. We hereby report genetically distinct $E$. coli isolates, belonging to seven STs, harboring the mcr-1 gene, associated to community and healthcare-acquired infections, and colonization in patients from three hospitals in Sao Paulo. These findings point out for the potential spread of plasmid-mediated colistin-resistance mechanism in E. coli strains in Brazil.

KEYWORDS: E. coli. Colistin-resistance. $m c r-1$. Community and healthcare-acquired infections.

\section{INTRODUCTION}

In the last decade, colistin-resistance among Enterobacteriacae has increased all over the world and nowadays it is a public health challenge $\mathrm{e}^{1,2}$. Until recently, colistinresistance was related to chromosome mutations ${ }^{1}$. Moreover, a novel plasmidmediated colistin-resistance gene, named $m c r$ - 1 , was identified in Escherichia coli isolates from animals and humans in China, in $2015^{2}$. Since then, several reports have described $m c r$ genes in animals bred for slaughter, from aquatic environments and in humans all over the world ${ }^{3-8}$. Soon afterwards, a study revealed travelers from South America colonized by E. coli harboring $m c r-1^{7}$. Of note, few studies have addressed the spread of $E$. coli harboring the $m c r-1$ gene in both, community and hospital settings. 
Our study described the clinical and molecular characteristics of community and healthcare-acquired infections as well as isolates from colonization caused by colistin-resistant $E$. coli carrying $m c r-1$ in three Brazilian hospitals.

\section{MATERIALS AND METHODS}

Colistin-resistant $E$. coli, from seven clinical samples, urine $(\mathrm{n}=5)$, feces $(\mathrm{n}=1)$, and surgical wound secretion $(\mathrm{n}=1)$, were identified in three high complexity hospitals, in Sao Paulo, Southeastern Brazil, between March, 2016 and January, 2018. Hospital A is a highly complex General Hospital with 800 beds, Hospital B is a tertiary General Hospital with 721 beds and Hospital C, with 170 beds, is specialized in cardiology and is predominantly surgical.

Data regarding demographic and clinical characteristics of all patients, including travels in the last year, contact with animals and rural area exposure, were retrieved from medical records, and an EPIINFO ${ }^{\circledR}$ database (CDC-USA 2017) was built. Clinical and laboratory data are stored at the Medical Research Laboratory LIM49, at Hospital das Clinicas. Feces positive for $E$. coli harboring $m c r-1$ were considered positive. This study was approved by the Ethics Committee of Hospital das Clinicas, University of Sao Paulo, Brazil (approval No 2.452.282).

The antimicrobial susceptibility test was performed using the Sensititre (Thermo Fisher Scientific, Cleveland, USA). The Minimum Inhibitory Concentration (MIC) for colistin was determined by the broth microdilution method as well ${ }^{8}$. MIC values $>2 \mathrm{mg} / \mathrm{L}$ were considered indicative of colistin-resistance, according to the Brazilian Committee on Antimicrobial Susceptibility Testing (BrCAST) ${ }^{9}$.

The pattern of resistance to polymyxins has been considered as suggestive that the isolate harbors the $m c r-1$ gene. The $m c r-1$ gene was screened using the Polymerase Chain Reaction (PCR) as described previously ${ }^{2}$ and confirmed by Sanger's sequencing within the NCBI database. The seven isolates were submitted to Whole Genome Sequencing (WGS) using the platforms MiSeq Illumina $^{\mathrm{TM}}$ (Illumina Inc., San Diego, CA, United States) ${ }^{10}$ or Ion Torrent (Thermo Fisher Scientific, Waltham, MA, USA) technologies ${ }^{11}$. De novo assemblies were carried out using SPAdes v.3.11.1 (Center for Algorithmic Biotechnology, St. Petersburg, Russia) ${ }^{12}$. The sequence type (ST) was checked by MLSTFinder tool (Multilocus Sequence Typing) and confirmed using the database PubMLST $^{13}$. Plasmids, resistance and virulence genes were analyzed using PlasmidFinder v. $2.1^{14}$, ResFinder v. $4.1^{15}$ and VirulenceFinder v. $2.0^{16}$ tools. The type of plasmids harboring the $m c r-1$ gene was determined by analysis on
Geneious v. R11 (University of California, CA, USA) ${ }^{17}$. The phylogenetic tree was constructed with the seven sequenced genomes based on SNPs using the REALPHY version 1.12 with default parameters ${ }^{18}$. Briefly, sequences were mapped to the reference sequence (E. coli ATCC25922) via Bowtie2. From this alignment, SNPs were calculated using Seaview ${ }^{19}$ and multiple sequence alignments were recreated using PhyML with 500 bootstrap replications for the tree construction ${ }^{18}$. The genome of the reference isolate was used as an outgroup to root the tree.

The conjugation experiment was performed with an streptomycin-resistant lactose-negative colistin-susceptible E. coli C600 strain as the recipient, in Luria Bertani broth medium. Transconjugant selection was executed on MacConkey agar plate with streptomycin $(2 \mathrm{mg} / \mathrm{mL})$ and colistin $(2 \mu \mathrm{g} / \mathrm{mL})$. Gene mobilization was confirmed by PCR.

\section{RESULTS}

The mean age of the patients was 64 years old (ranged from 3 to 91 years old) and five of the seven were female. Most patients presented with urinary tract infections (UTI) (5/7) (Table 1). Four of five UTI strains were identified within $24 \mathrm{~h}$ of admission. Only one patient had a history of exposure to a rural area, with breeding of animals and consumption of meat. Two others had no history of exposure to the countryside or travel in the last year (Table 1). Although five strains were from hospital B, the patients were not from the same family, neither had epidemiological links. Patients were on contact precaution until hospital discharge.

Each of the seven E. coli isolates belonged to a different ST (ST744, ST131, ST69, ST48, ST354, ST57, ST10) (Figure 1). In total, 163,155 SNPs were analyzed in the phylogenetic tree and the number of SNPs is identified in each node (Figure 1). No cluster pattern was observed with the exception of isolates from hospital A and C (ST44 and ST10) that grouped closer with 5,104 SNPs of difference (Figure 1).

The isolates differed regarding the resistome varying up to nine antimicrobials to which isolates were resistant (Table 1). The most relevant mechanisms of resistance detected were genes that encode betalactamases $\left(b l a_{\text {TEM-IB }}\right.$; $\left.b l a_{\text {OХA-1 }} ; b l a_{\text {СMY-2}} ; b l a_{\text {СТХ-M-2 }} ; b l a_{\text {СTX-M-8 } 8}\right)$, aminoglycosides modifying enzymes (aadA1; aadA2; aadB; aac(3)-IId; aac(3)-VIa; aph(3')-Ia; aac(6')Ib-cr; aadA5; aph(6)-Id; $\left.a p h\left(3^{\prime \prime}\right)-I b\right)$, and genes that can lead to resistance to macrolides (mph $(A))$, chloramphenicol (catAl; catB4; floR), sulfonamides (sull; sul2; sul3); tetracyclines (tet $(A)$; tet( $(B))$ and quinolones (qnrSl). 


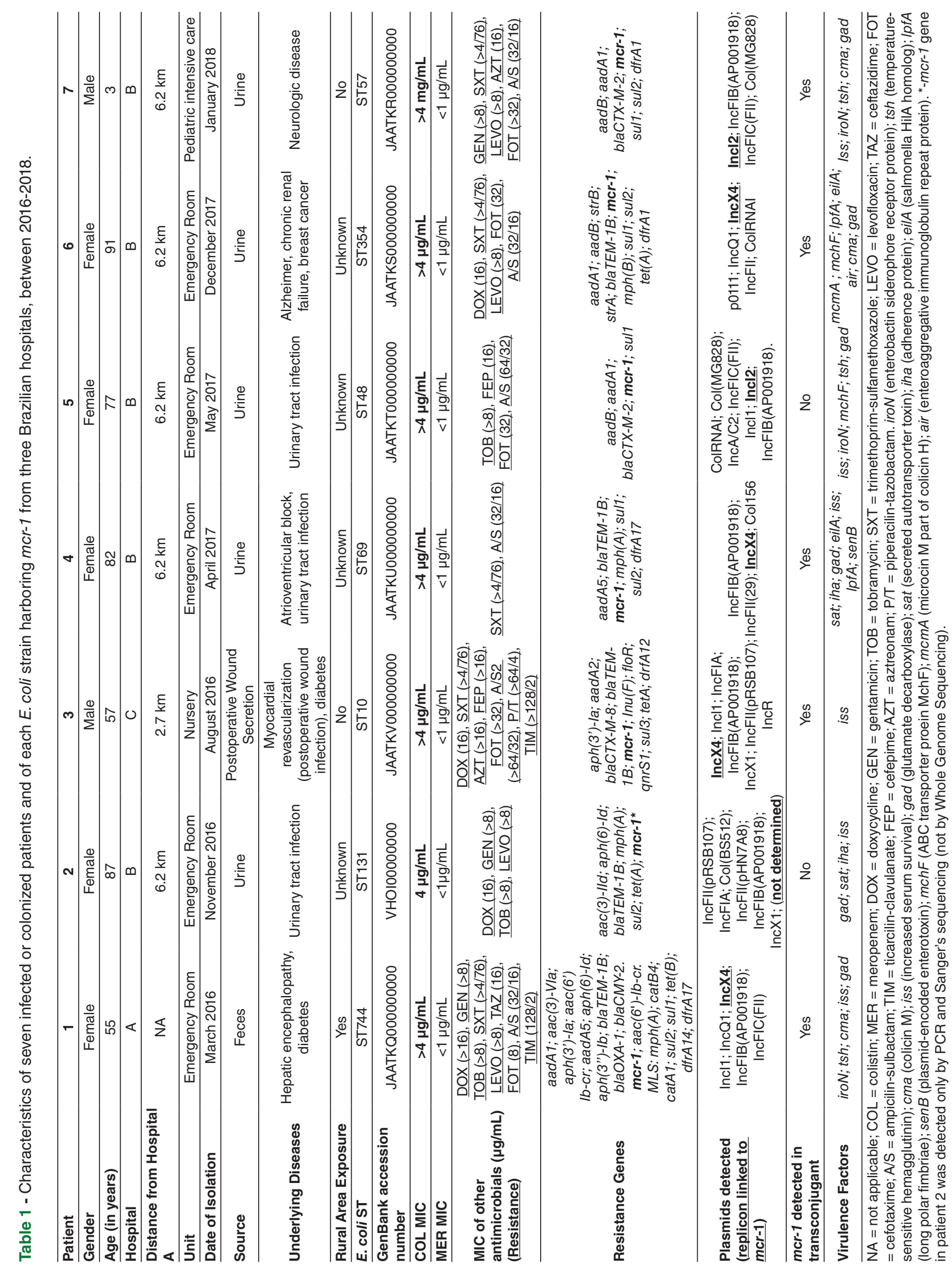




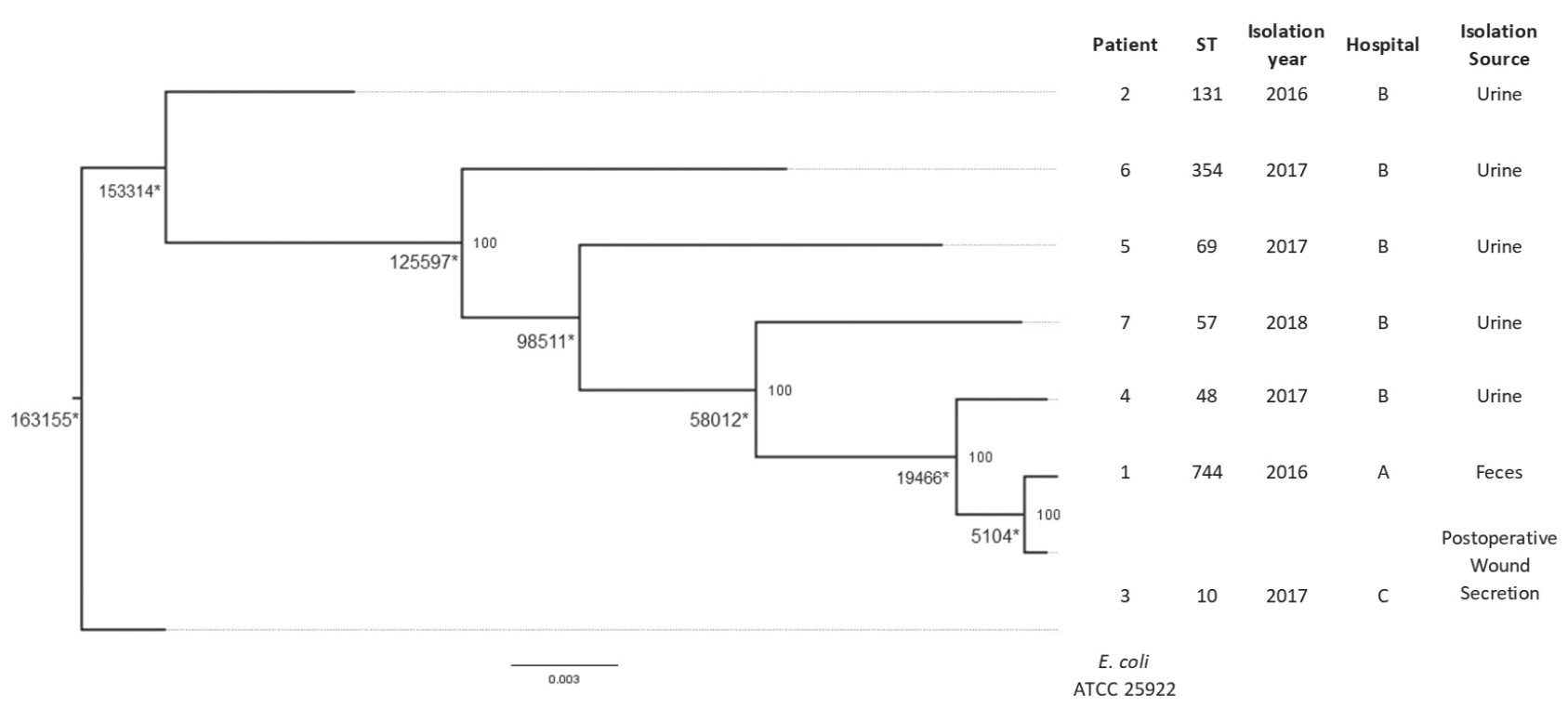

Figure 1 - Maximum likelihood phylogenetic tree of the E. coli genomes. The tree was rooted using the reference genome ATCC25922. SNPs are identified in each node with an asterisk $\left({ }^{*}\right)$.

In isolates from patients 1, 3 and 6, mcr-1 and IncX4 replication initiation genes were identified in the same contigs, and from patient 4 and patient 7 with IncI2 replication gene. The isolate from patient 5 presented with $m c r-1$ and replication initiation genes in different contigs. Nevertheless, the $m c r-1$ gene was detected on the transconjugant of the isolate from patient 5, a type of carrier plasmid that could not be determined by WGS. Transference of $m c r-1$ by conjugation was possible in five of the seven isolates, the lactose-negative E. coli C600 transconjugants were streptomycin-resistant (as expected), but also colistin-resistant (Table 1). The $m c r-1$ gene in the isolate from patient 2 was detected only by PCR and confirmed by Sanger's sequencing, but not by WGS, which could be due to the loss of gene or an impairment of the sequencing platform, as it was the only isolate sequenced by the Ion Torrent technology.

\section{DISCUSSION}

We tested phenotypically and genotypically seven isolates of colistin-resistant $E$. coli from seven different colonized or infected patients from three Brazilian hospitals. The isolates have other antimicrobial resistance genes, among which genes encoding extended-spectrum

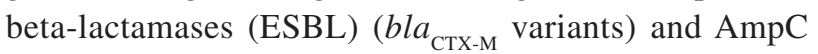
cephalosporinase $\left(b l a_{\mathrm{CMY}}\right)$ are frequently transmitted by conjugation.

Among the seven different STs, six have already been assigned to isolates harboring $m c r-1$ in other countries ${ }^{6,20-22}$. We detected $m c r-1$ in IncX4 plasmids of the isolates from four patients (Table 1), similar to some previously reported in Brazil ${ }^{23,24}$, and in Incl2 plasmids of the isolates from two patients (Table 1), similar to the first isolate described in China ${ }^{2}$. Transference by conjugation was not possible with isolates from patients 2 and 4 , which could be related to carriage by plasmids not as easily transferable as the IncX4 type or impairment in transfer mechanisms. In fact, a previous study reported this challenge, as the $m c r-1$ gene was successfully transferred to E. coli strain C600 only in $34 \%$ of the studied isolates ${ }^{25}$.

Resistance to colistin usually rises from selective pressure due to the use of polymyxins ${ }^{2}$. In Brazil, colistin was widely used as a growth promoter in animal production until 2016, when it was banned ${ }^{26}$ due to identification of plasmid-mediated colistin-resistant isolates in the country. It is important to point out that the prevalence of colistinresistance isolates can be underestimated, as colistinsusceptibility is not routinely tested in outpatients' clinical samples.

Up to now, the $m c r-1$ gene is the most frequent plasmidmediated colistin-resistance gene reported in Brazil ${ }^{26,27}$. It has been described in Salmonella enterica isolated from poultry meat ${ }^{21}$, from food ${ }^{27}$, and in E. coli from cattle and humans ${ }^{28,29}$ and Klebsiella pneumoniae from humans ${ }^{29}$.

At first, susceptibility to antimicrobials besides polymyxins was frequently observed in isolates harboring the $m c r-1$ gene. However, the reports of isolates with resistance mechanisms to other antimicrobials, such as cephalosporins and carbapenems, are increasing ${ }^{24,28}$. In our study, all the strains were susceptible to carbapenem, but carried genes encoding other beta-lactamases ( bla $_{\text {OXA }}$, $b l a_{\mathrm{TEM}}, b l a_{\mathrm{CTX}-\mathrm{M}}$ and $b l a_{\mathrm{CMY}}$ ), frequently transmitted by conjugation $^{28}$ as well as aminoglycosides modifying 
enzymes (aadA1; aadA2; aadB; aac(3)-IId; aac(3)-VIa; aph(3')-Ia; aac(6')Ib-cr; aadA5; aph(6)-Id; aph(3')-Ib), and genes that can lead to resistance to macrolides $(m p h(A))$, chloramphenicol (catA1; catB4; floR), sulfonamides (sull; sul2; sul3); tetracyclines $(\operatorname{tet}(A) ; \operatorname{tet}(B))$ and quinolones (qnrSl)

The SNPs tree and the seven STs described showed the diversity of E. coli harboring $m c r-1$ in Brazil. Horizontal $m c r-1$ gene transfer is supported by the SNP analysis as the strains were not clonal, as expected from different STs. Moreover, they were not closely related in regard of the hospital which they were isolated from or by year, supporting their acquisition from different sources and/or periods. Also, these results highlight the potential of colistin-resistance horizontal dissemination, and the possible spread of different STs capable of harboring plasmids with $m c r-1$ in the community, since five isolates were collected in emergence departments, and four were identified within 24 hours of admission. However, acquisition in previous hospitalizations cannot be discarded. Therefore, our findings alert for the potential dissemination of $\mathrm{mcr}-1$ in the country and emphasize the need to rethink strategies to control the colistin-resistance, such as surveillance for E. coli strains, screening for $\mathrm{mcr}-1$ at emergency departments and in the food chain.

An important limitation of our study was that, although, the $m c r-1$ of isolate 2 was detected by PCR and confirmed by Sanger's sequencing, it was not found using the IonTorrent platform. It will be important to confirm our finding using a different new generation sequencing platform such as Illumina.

In summary, we reported genetically distinct $E$. coli isolates harboring the $\mathrm{mcr}-1$ gene associated to communityand healthcare-acquired infections and colonization in patients from three hospitals in Sao Paulo. The strains belonged to different STs. Our findings pointed out for the potential dissemination of this plasmid-mediated colistinresistance mechanism in E. coli strains.

\section{ACKNOWLEDGMENTS}

We thank the laboratory personnel who contributed to the study.

\section{REFERENCES}

1. Moffatt JH, Harper M, Boyce JD. Mechanisms of polymyxin resistance. Adv Exp Med Biol. 2019;1145:55-71.

2. Liu YY, Wang Y, Walsh TR, Yi LX, Zhang R, Spencer J, et al. Emergence of plasmid-mediated colistin resistance mechanism mcr-1 in animals and human beings in China: a microbiological and molecular biological study. Lancet Infect Dis. 2016;16:161-8

3. Wu C, Wang Y, Shi X, Wang S, Ren H, Shen Z, et al. Rapid rise of the ESBL and mcr-1 genes in Escherichia coli of chicken origin in China, 2008-2014. Emerg Microbes Infect. 2018;7:30.

4. Cannatelli A, Giani T, Antonelli A, Principe L, Luzzaro F, Rossolin GM. First detection of the mcr-1 colistin resistance gene in Escherichia coli in Italy. Antimicrob Agents Chemother. 2016;60:3257-8.

5. Hu YY, Wang YL, Sun QL, Huang ZX, Wang HY, Zhang R, et al. Colistin resistance gene mcr-1 in gut flora of children, Int J Antimicrob Agents. 2017;50:593-7.

6. Hadjadj L, Riziki T, Zhu Y, Li J, Diene SM, Rolain JM, et al. Study of mcr-1 gene-mediated colistin resistance in enterobacteriaceae isolated from humans and animals in different countries. Genes (Basel). 2017;8:394.

7. Fernandes MR, Moura Q, Sartori L, Silva KC, Cunha MP, Esposito $\mathrm{F}$, et al. Silent dissemination of colistin-resistant Escherichia coli in South America could contribute to the global spread of the mcr-1 gene. Euro Surveill. 2016;21:1-6.

8. Matuschek E, Åhman J, Webster C, Kahlmeter G. Antimicrobial susceptibility testing of colistin: evaluation of seven commercial MIC products against standard broth microdilution for Escherichia coli, Klebsiella pneumoniae, Pseudomonas aeruginosa, and Acinetobacter spp. Clin Microbiol Infect. 2018;24:865-70.

9. Brazilian Committee on Antimicrobial Susceptibility Testing. [cited 2021 Apr 5]. Available from: http://brcast.org.br/

10. Benjak A, Sala C, Hartkoorn RC. Whole-genome sequencing for comparative genomics and de novo genome assembly. Methods Mol Biol. 2015;1285:1-16

11. Baez-Ortega A, Lorenzo-Diaz F, Hernandez M, Gonzalez-Vila CI, Roda-Garcia JL, Colebrook M, et al. IonGAP: integrative bacterial genome analysis for Ion Torrent sequence data. Bioinformatics. 2015;31:2870-3.

12. Bankevich A, Nurk S, Antipov D, Gurevich AA, Dvorkin M, Kulikov AS, et al. SPAdes: a new genome assembly algorithm and its applications to single-cell sequencing. J Comput Biol. 2012;19:455-77.

13. Jolley KA, Bray JE, Maiden MC. Open-access bacterial population genomics: BIGSdb software, the PubMLST.org website and their applications. Wellcome Open Res. 2018;3:124.

14. Carattoli A, Zankari E, García-Fernández A, Larsen MV, Lund $\mathrm{O}$, et al. In silico detection and typing of plasmids using PlasmidFinder and plasmid multilocus sequence typing. Antimicrob Agents Chemother. 2014;58:3895-903.

15. Zankari E, Hasman H, Kaas RS, Seyfarth AM, Agers $\varnothing$ Y, Lund $\mathrm{O}$, et al. Genotyping using whole-genome sequencing is a realistic alternative to surveillance based on phenotypic antimicrobial susceptibility testing. J Antimicrob Chemother. 2013;68:771-7. 
16. Joensen KG, Scheutz F, Lund O, Hasman H, Kaas RS, Nielsen $\mathrm{EM}$, et al. Real-time whole-genome sequencing for routine typing, surveillance, and outbreak detection of verotoxigenic Escherichia coli. J Clin Micobiol. 2014;52:1501-10.

17. Kearse M, Moir R, Wilson A, Stones-Havas S, Cheung M, Sturrock S, et al. Geneious Basic: an integrated and extendable desktop software platform for the organization and analysis of sequence data. Bioinformatics. 2012;28:1647-9.

18. Bertels F, Silander OK, Pachkov M, Rainey PB, van Nimwegen E. Automated reconstruction of whole-genome phylogenies from short-sequence reads. Mol Biol Evol. 2014;31:1077-88.

19. Galtier N, Gouy M, Gautier C. SEAVIEW and PHYLO_WIN: two graphic tools for sequence alignment and molecular phylogeny Comput Appl Biosci. 1996;12:543-8.

20. Ben Sallem R, Ben Slama K, Rojo-Bezares B, Porres-Osante N, Jouini A, Klibi N, et al. IncI1 plasmids carrying bla CTX-M-1 or bla CMY-2 genes in Escherichia coli from healthy humans and animals in Tunisia, Microb Drug Resist. 2014;20:495-500

21. Jørgensen B, Søraas A, Arnesen LS, Leegaard T, Sundsfjord A, Jenum PA. First environmental sample containing plasmidmediated colistin-resistant ESBL-producing Escherichia coli detected in Norway. APMIS. 2017;125:822-5.

22. Corbella M, Mariani B, Ferrari C, Comandatore F, Scaltriti E Marone $\mathrm{P}$, et al. Three cases of mcr-1-positive colistin-resistant Escherichia coli bloodstream infections in Italy, August 2016 to January 2017. Eurosurveillance. 2017;22:30517.

23. Moreno LZ, Gomes VT, Moreira J, Oliveira CH, Peres BP, Silva AP, et al. First report of mcr-1-harboring Salmonella enterica serovar Schwarzengrund isolated from poultry meat in Brazil, Diagn Microbiol Infect Dis. 2019;93:376-9.

24. Dalmolin TV, Martins AF, Zavascki AP, Lima-Morales D, Barth $\mathrm{AL}$. Acquisition of the mcr-1 gene by a high-risk clone of KPC-2-producing Klebsiella pneumoniae ST437/CC258, Brazil. Diagn Microbiol Infect Dis. 2018;90:132-3.

25. Wang Y, Tian GB, Zhang R, Shen Y, Tyrrell JM, Huang X, et al. Prevalence, risk factors, outcomes, and molecular epidemiology of mcr-1-positive Enterobacteriaceae in patients and healthy adults from China: an epidemiological and clinical study. Lancet Infect Dis. 2017;17:390-9.

26. Pontes LS, Pimenta R, Silveira MC, Tavares-Teixeira CB, Pereira $\mathrm{NF}$, Conceição Neto OC, et al. Escherichia fergusonii harboring IncHI2 plasmid containing mor-1 Gene: a novel reservoir for Colistin resistance in Brazil. Microb Drug Resist. 2020 In Press.

27. Rau RB, Lima-Morales D, Wink PL, Ribeiro AR, Barth Al. Salmonella enterica mcr-1 positive from food in Brazil: detection and characterization. Foodborne Pathog Dis. 2020;17:202-8.

28. Palmeira JD, Ferreira H, Madec JY, Haenni M. Draft genome of a ST443 mcr-1- and bla ${ }_{\text {CTX-M-2 }}$-carrying Escherichia coli from cattle in Brazil. J Glob Antimicrob Resist. 2018;13:269-70.

29. Perdigão Neto LV, Corscadden L, Martins RC, Nagano DS, Cunha MP, Neves PR, et al. Simultaneous colonization by Escherichia coli and Klebsiella pneumoniae harboring mcr-1 in Brazil. Infection. 2019;47:661-4. 